\title{
Establishment and comparison of two reliable hyperkinetic pulmonary hypertension models in rabbits
}

\author{
Chuanzhen Liu, MD, ${ }^{\mathrm{a}}$ Zhibo Yan, MD, PhD, ${ }^{\mathrm{b}}$ Changcun Fang, MD, PhD, ${ }^{\mathrm{a}}$ Guangqing Cao, MD, PhD, ${ }^{\mathrm{a}}$ \\ Biao Wang, MD, PhD, ${ }^{a}$ Sijie Li, MD, ${ }^{a}$ and Shuming $\mathrm{Wu}, \mathrm{MD}, \mathrm{PhD}^{\mathrm{a}}$
}

Objectives: We sought to explore and create a reliable, convenient, and economic hyperkinetic pulmonary artery hypertension (PAH) model and confirm the exact time of establishing a reversible or irreversible model to serve as a platform for future studies.

\begin{abstract}
Methods: We used a common carotid artery and jugular vein shunt with an anastomosis and cuff to create a hyperkinetic PAH model in rabbits. At 1, 2, 3, 6, and 12 months postoperatively, the systolic pressure, mean pulmonary arterial pressure, and mean arterial pressure were measured by catheterization and the right ventricle hypertrophy index was calculated. Pathologic changes in the small pulmonary arteries were observed with hematoxylin and eosin staining, and the Heath-Edwards classification system was used to evaluate PAH.
\end{abstract}

Results: The anastomosis and cuff graft groups both increased in systolic pressure, mean pulmonary arterial pressure $(P<.05)$, and right ventricle hypertrophy index $(P<.05)$. However, the anastomosis method resulted in a lower mortality rate, greater patency, and overall success rate compared with the cuff graft method $(P<.05)$. Furthermore, from the observed pathologic changes and the Heath-Edwards classification system, a reversible and an irreversible PAH model was established at 3 and 6 months postoperatively, respectively.

Conclusions: The common carotid artery and jugular vein anastomosis method is a stable hyperkinetic PAH model in rabbits. Reversible and irreversible PAH models were established at 3 and 6 months postoperatively, respectively. (J Thorac Cardiovasc Surg 2014;148:2353-9)

Pulmonary arterial hypertension (PAH) is a clinical pathophysiologic syndrome characterized by a progressively increased pulmonary vascular resistance that can be caused by heterogeneous disease and/or other pathologic abnormalities. The clinical manifestations include increased right ventricular afterload, decreased activity intolerance, and eventual death resulting from right heart failure. ${ }^{1,2}$ PAH is characterized by pulmonary vasoconstriction, aberrant proliferation of smooth muscle and endothelial cells, and, later, irreversible increased pulmonary vascular resistance. ${ }^{3}$ However, the etiology of PAH is not completely understood.

PAH is a significant contributor to the morbidity and mortality in patients with congenital heart disease, especially in those with Eisenmenger syndrome. ${ }^{4}$ Thus, it is imperative to establish a pulmonary arterial hypertension model to study PAH. Currently, a number of methods are available

\footnotetext{
From the Departments of Cardiovascular Surgery ${ }^{\mathrm{a}}$ and General Surgery, ${ }^{\mathrm{b}}$ Qilu Hospital of Shandong University, Jinan, Shandong, People's Republic of China. This work was supported by the Shandong Provincial Natural Science Foundation of China (grant ZR2011HM025)

Disclosures: Authors have nothing to disclose with regard to commercial support.

Received for publication Oct 8, 2013; revisions received Nov 17, 2013; accepted for publication Dec 5, 2013; available ahead of print Feb 9, 2014.

Address for reprints: Shuming Wu, MD, PhD, Department of Cardiovascular Surgery, Qilu Hospital of Shandong University, 107\#, Wenhua Xi Rd, Jinan, Shandong 250012, People's Republic of China (E-mail: shumingwu001@126.com). $0022-5223 / \$ 36.00$

Copyright $(2014$ by The American Association for Thoracic Surgery http://dx.doi.org/10.1016/j.jtcvs.2013.12.066
}

to induce PAH, including subcutaneous injection of monocrotaline,${ }^{5-7}$ chronic hypoxia,${ }^{8,9}$ gene knockouts, ${ }^{10}$ vascular endothelial growth factor receptor blockade ${ }^{11}$ arteriovenous shunts, ${ }^{12}$ and other newer methods. ${ }^{13-16}$ However, only arteriovenous shunt methods will result in the same pathophysiologic changes present during congenital heart disease in humans.

We have previously attempted to establish new methods to mimic PAH in both rabbits and rodents, with limited success. ${ }^{17,18}$ We first established a PAH model ${ }^{17}$ using a left common carotid artery and pulmonary trunk anastomosis in rabbits and later established another model, ${ }^{18}$ with a left common carotid artery and left jugular vein shunt in a cuff style in Wistar rats. However, the first model was complicated and required thoracotomy, which has a potentially high mortality rate. However, the long-term patency rate of the second model was questionable. Furthermore, the exact period required to successfully establish an irreversible PAH model after arteriovenous shunt was unknown. PAH results in pathologic changes that are initially reversible but become irreversible. Thus, when a PAH model has been established, it is essential to determine the point at which both reversible and irreversible stages occur to facilitate future studies that will more accurately reflect PAH in humans.

Our goal was to establish a convenient, economic, highly successful, and stable PAH model that better represents the aberrant hemodynamic state in children with congenital heart disease. We also sought to determine the point at 


\author{
Abbreviations and Acronyms \\ CJSA $=$ common carotid artery and jugular vein \\ shunt by anastomosis \\ CJSC $=$ common carotid artery and jugular vein \\ shunt by cuff style method \\ PAH = pulmonary artery hypertension \\ $\mathrm{RV} \quad=$ right ventricle \\ $\mathrm{LV}+\mathrm{S}=$ left ventricle plus septum
}

which the reversible and irreversible stages of PAH occur to facilitate the design of future studies.

\section{METHODS \\ Animals}

A total 120 male New Zealand white rabbits (age, 4 weeks; weight, 545 $\pm 27 \mathrm{~g}$ ) were provided by the Laboratory Animal Center of Shandong University. The rabbits received humane care, and the experiments were performed in accordance with the guidelines of Animal Care and Use Committee of Shandong University. The rabbits were housed individually in a room at a constant ambient temperature and humidity with a 12-hour light and dark cycle. After 10 days of acclimation, the 120 rabbits were randomly assigned into the following 3 groups (40 rabbits each): group 1 , common carotid artery and jugular vein shunt by anastomosis (CJSA); group 2, common carotid artery and jugular vein shunt in cuff style method (CJSC) group; and group 3, sham operation group.

\section{Surgical Procedures}

CJSA group. In the CJSA group of 40 rabbits, the rabbits were anesthetized with an intravenous injection of pentobarbital sodium $(30 \mathrm{mg} / \mathrm{kg})$. Once the anesthesia took effect, a 3-cm midline incision was made in the neck. The right external jugular vein was exposed and then injected with $1 \mathrm{mg} / \mathrm{kg}$ heparin. The right common carotid artery and right internal jugular vein were carefully dissected. The right common carotid artery was clamped at the proximal site and ligated at the distal site. The right common carotid artery was then amputated immediately before the bifurcation. Under an operative microscope, a 2-mm hole was made in the right internal jugular vein and an end-to-side anastomosis was made between the right common carotid artery and the right internal jugular vein using Prolene 7-0 suture (Figures 1 and 2). The degree of anastomosis patency was tested by releasing the vascular clamps to visualize the pulsative bright red blood flow in the proximal site of the right internal jugular vein. Penicillin was administered postoperatively to prevent secondary infection.

CJSC group. CJSC was performed in a fashion similar to that for the CJSA group. However, the end-to-side shunt was made by a 5 -mm-long polyvinyl tube with a 2-mm external diameter and 1.8- $\mathrm{mm}$ internal diameter. The carotid artery was inserted into the tube, and the intimal side of the artery was everted and ligated using a 2-0 thin thread. Finally, the cuff-mounted carotid artery was inserted into the left jugular vein through a 2-mm incision and ligated to connect these 2 vessels, endothelium to endothelium $^{18,19}$ (Figures 2 and 3).

Sham operation group. The vessels were isolated as detailed for the CJSA and CJSC groups. The sham operation was performed by isolating the vessels without an additional surgical procedure.

Aspirin $(10 \mathrm{mg} / \mathrm{kg} / \mathrm{d})$ was administrated to the rabbits from all groups to prevent coagulation that continued to the end of experimental period. Rabbits from each group were killed and evaluated 1, 2, 3, 6, and 12 months after surgery. Data collected from the rabbits with failed shunts or those that died of the surgery were excluded from additional analysis.

\section{Data Acquisition and Analysis}

To detect shunt patency and overall cardiac function, echocardiography was performed after anesthesia and before death. As stated, all the data from rabbits that died as a result of surgery or had failed shunts (occluded shunts or occluded anastomoses) were excluded.

The systolic pulmonary artery pressure, mean pulmonary artery pressure, and mean arterial pressure were measured by catheterization. These hemodynamic data were collected by first anesthetizing the rabbits and exposing the left jugular vein. A $3 \mathrm{~F}$ polyethylene catheter was then inserted into the vein and advanced into the right ventricular and then the main pulmonary artery under fluoroscopic guidance. In addition, the position of the catheter was confirmed by transitional changes in the pressure curve displayed on the monitor. The systemic pressure was monitored by a second catheter inserted into the left common carotid artery. Both of the catheters were connected to a transducer, and the pulmonary arterial pressure and systemic pressure were recorded. The hemodynamic data were recorded before and after closure of the shunt vessels. The parameters were recorded 3 times for each rabbit, and the average pressure was calculated.

After collection of the hemodynamic data, the rabbits were killed by a pentobarbital sodium overdose. The heart tissue was collected and separated into right ventricle (RV) and left ventricle plus septum $(\mathrm{LV}+\mathrm{S})$. The extent of right ventricular hypertrophy was evaluated by the ratio of the $\mathrm{RV}$ mass to the $\mathrm{LV}+\mathrm{S}$ mass.

The rabbit lungs were fixed in $4 \%$ paraformaldehyde in phosphatebuffered saline for morphology analysis. The lungs were subjected to a dehydration series, embedded in paraffin, and sectioned in $5-\mu \mathrm{m}$ slices. Next, the slices were stained with hematoxylin-eosin and examined under an optical microscope (Olympus BX51, Olympus, Center Valley, Pa) to assess the changes occurring in the small arteries.

\section{Statistical Analysis}

The operative time, hemodynamic data, and right ventricular hypertrophy data are expressed as the mean \pm standard deviation. The successful rate was analyzed using the chi-square test. For rabbits with shunts, the data collected before and after shunt closure were compared using Student's $t$ tests. Analysis of variance was used to test for differences among the CJSC, CJSA, and sham operation groups. The Statistical Package for Social Sciences, version 17.0, for Windows (SPSS, Chicago, Ill), was used for all statistical analyses.

\section{RESULTS}

The mean operative time in the CJSC and CJSA groups was $1.40 \pm 0.12$ hours and $1.48 \pm 0.21$ hours, respectively (Table 1). No significant difference was found in the final body weight. The mortality rate in the CJSC group (27.5\%) was greater than that in the CJSA group $(12.5 \%)$. In the CJSC group, 3 rabbits died of a common carotid artery injury, 6 died of cuff drop off and hemorrhage within 24 hours of surgery, and 2 died of undefined complications (likely heart failure). In the CJSA group, 4 rabbits died of a common carotid artery injury and uncontrolled bleeding, and only 1 died of undefined complications. According to the echocardiographic findings and the dissection before death, 7 of the 29 surviving rabbits in the CJSC group and 3 of 35 surviving rabbits in the CJSA group presented with cuff occlusion.

\section{Pulmonary Hemodynamics}

To exclude the influence of the anastomosis and cuff on the pulmonary artery pressure, the pulmonary artery 


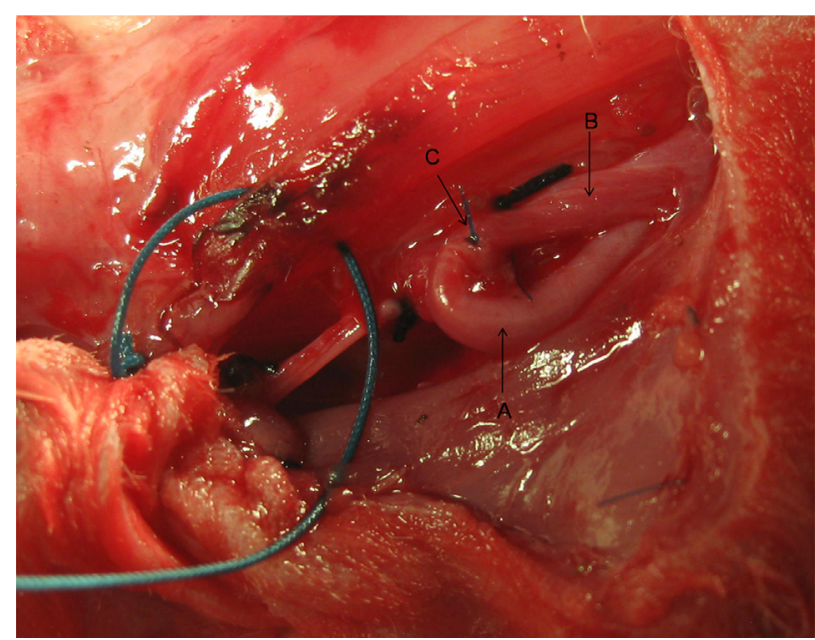

FIGURE 1. End-to-side anastomosis (C) between the right common carotid artery (A) and right internal jugular vein (B).

pressure was measured before and after closure of the shunt vessels. Compared with the sham operation group, the CJSC and CJSA groups had a greater systolic pulmonary arterial pressure and mean pulmonary arterial pressure before closure. Although the pulmonary artery pressure decreased after closure in the sham operation group (Figure 4), the systolic pulmonary arterial pressure and mean pulmonary arterial pressure were maintained at a greater value in the CJSC and CJSA groups $(P<.05$; Figure 5). When we compared the CJSC and CJSA groups, no significant differences were observed.

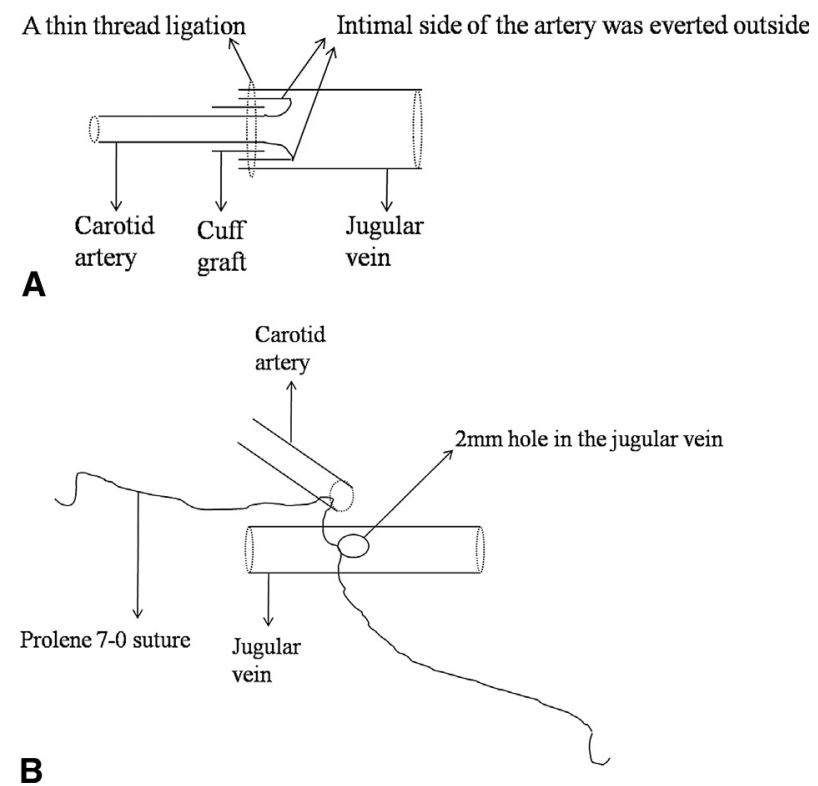

FIGURE 2. Drawing of surgical techniques and differences between (A) common carotid artery and jugular vein shunt by cuff style method (CJSC) and (B) common carotid artery and jugular vein shunt by anastomosis (CJSA).

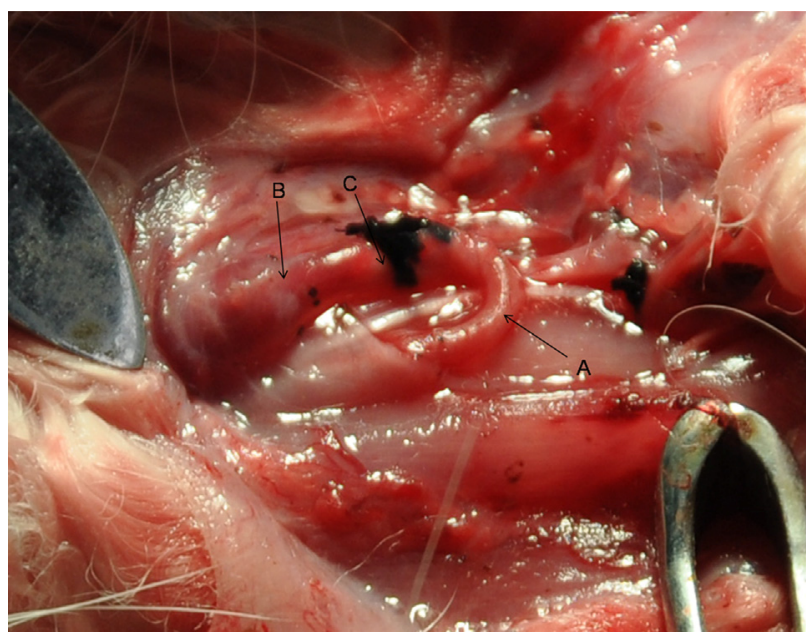

FIGURE 3. Right common carotid artery (A) and right internal jugular vein (B) shunt in cuff style (C).

\section{Right Ventricular Hypertrophy}

A significant elevation in the ratio of the $\mathrm{RV}$ mass to the $\mathrm{LV}+\mathrm{S}$ mass was found in the CJSC and CJSA groups compared with the sham operation group $(P<.05$; Figure 6). However, no differences were found between the CJSC and CJSA groups.

\section{Morphologic Findings}

The pathologic features of hypertensive pulmonary vascular disease was classified according to the HeathEdwards classification system ${ }^{20}$ (Table 2). Within 3 months, the rabbit lung microvascular vessel in the CJSA and CJSC groups demonstrated uniform intimal thickening and medial hypertrophy, with degeneration, swelling, hyperplasia, and fibrosis of nonmuscular arterial endothelial cells. Furthermore, these groups had developed smooth muscle tissue and hyperplasia of the elastic collagen fibers (grade I-II) in the tunica media (Figure 7, B). Moreover, a severe narrowing or occlusion of the lumen was frequently found in the CJSC (4 of 22) and CJSA (11 of 32) groups (grade III; Figure 7, C). By 3 months, 2 of 12 in the CJSC group and 4 of 20 in the CJSA group demonstrated plexiform lesions in the lung microvascular vessels (grade IV-VI), which marked an irreversible pathologic change

TABLE 1. Operative time and success rate

\begin{tabular}{lccccc}
\hline Group & Rabbits & $\begin{array}{c}\text { Operative } \\
\text { time (h) }\end{array}$ & Died (n) & Occlusion (n) & $\begin{array}{c}\text { Success } \\
\text { rate (\%) }\end{array}$ \\
\hline CJSC & 40 & $1.40 \pm 0.12$ & 11 & 7 & 55 \\
CJSA & 40 & $1.48 \pm 0.21$ & 5 & 3 & $80^{*}$ \\
\hline
\end{tabular}

No significant difference was found in operative time, and the CJSA group had a greater success rate than the CJSC group. CJSC, Common carotid artery and jugular vein shunt by cuff style method; CJSA, common carotid artery and jugular vein shun by anastomosis. ${ }^{*} P<.05$ versus CJSC group. 
SPAP before and after closure in CJSC group

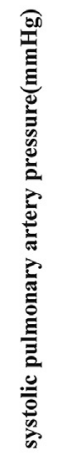

A

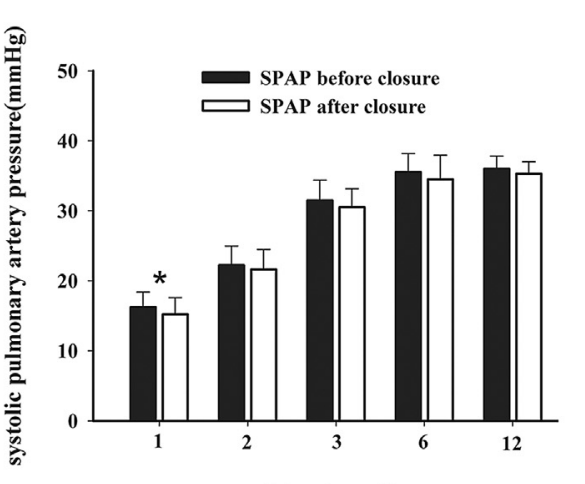

Time(month)

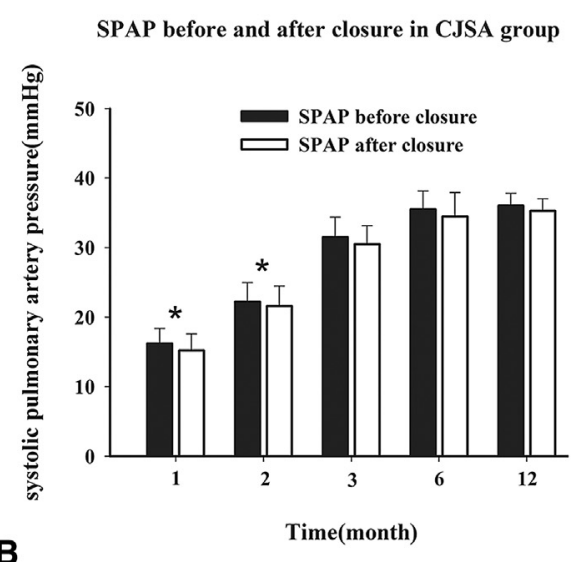

B

FIGURE 4. Systolic pulmonary arterial pressure (SPAP) before and after closure of shunt vessels in (A) the common carotid artery and jugular vein shunt by cuff style method (CJSC) group and (B) the common carotid artery and jugular vein shunt by anastomosis (CJSA) group. In the CJSC group, the SPAP before closure was greater than that after closure in 1 month, with no significant difference after 2 months $(* P<.05)$. In the CJSA group, the SPAP before closure was greater than that after closure at 1 and 2 months, with no significant difference after 3 months $(* P<.05)$.

(Figure 7,D), consistent with the irreversible changes that occur in human PAH. However, no such pathologic changes were seen before 3 months in either group.

\section{DISCUSSION}

In the present report, we have described 2 reliable hyperkinetic PAH models in rabbits. The hemodynamic data,
SPAP

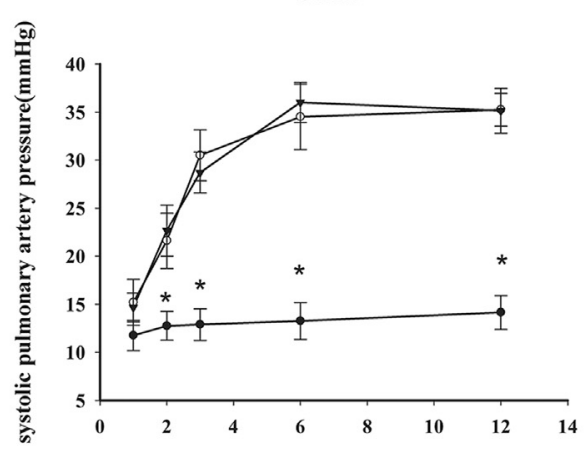

A

Time(month)

MAP

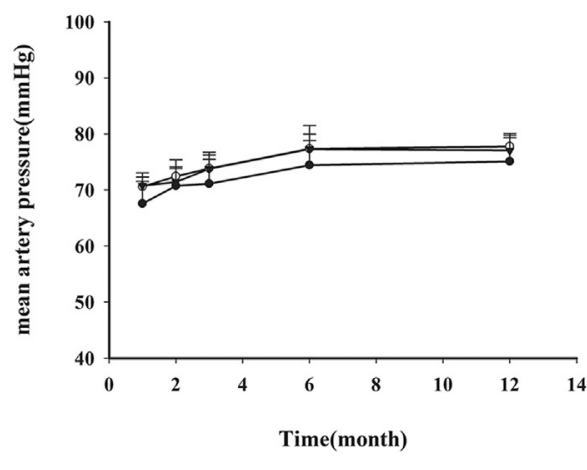

MPAP

B

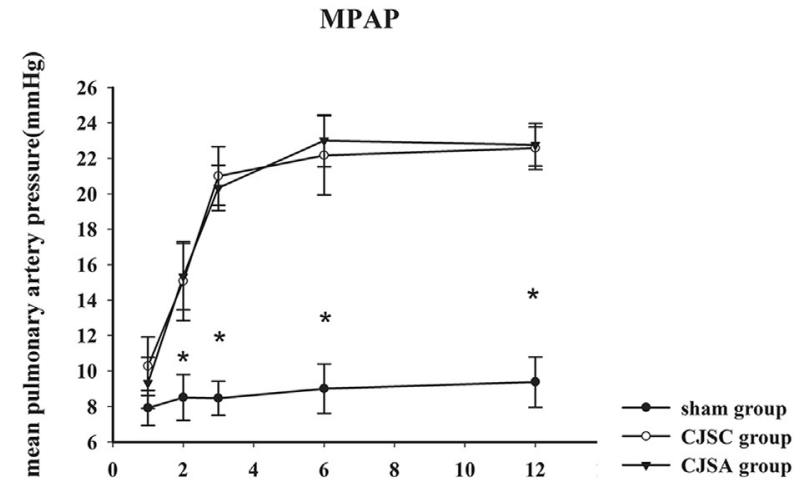

Time(month)

FIGURE 5. The systolic pulmonary arterial pressure ( $S P A P)$, mean pulmonary arterial pressure $(M P A P)$, and mean arterial pressure (MAP) after closure of shunt vessels. A and B, Compared with the sham operation group, the common carotid artery and jugular vein shunt by cuff style method (CJSC) group and common carotid artery and jugular vein shunt by anastomosis (CJSA) group showed greater SPAP and MPAP. Both SPAP and MPAP were increased at 6 months preoperatively and remained steady from 6 to 12 months $(* P<.05)$. C, Compared with the sham operation group, no significant difference was found in the MAP between the CJSC and CJSA groups. 


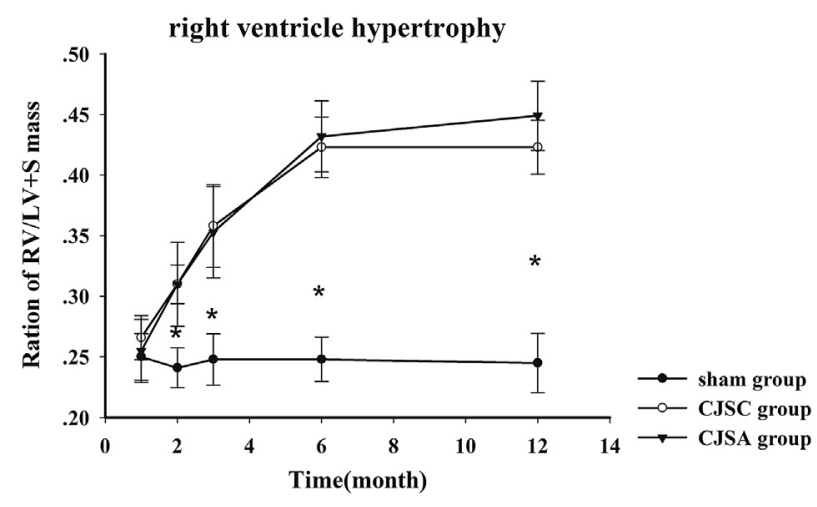

FIGURE 6. Right ventricular hypertrophy. Compared with the sham operation group, the common carotid artery and jugular vein shunt by cuff style method (CJSC) group and common carotid artery and jugular vein shunt by anastomosis (CJSA) group showed a greater ratio of the right ventricular mass to the left ventricular plus septum mass $(R V / L V+S)\left({ }^{*} P<.05\right)$.

right ventricular hypertrophy index (ratio of the RV mass to the $\mathrm{LV}+\mathrm{S}$ mass), echocardiographic findings, and histopathologic findings were compared between the 2 models. Because the mortality and patency rate are significant influencing factors in establishing a successful model, these 2 factors were also evaluated. Additionally, we examined and characterized the histopathologic changes that mediate the transition from reversible to irreversible stages of PAH. We successfully determined the period that elapsed before establishing an irreversible model.

TABLE 2. Number of rabbits according to Heath-Edwards classification

\begin{tabular}{lccc}
\hline $\begin{array}{c}\text { Postoperative point } \\
\text { at which rabbits were } \\
\text { killed (no. killed) }\end{array}$ & Grade I-II & Grade III & Grade IV-VI \\
\hline CJSC & & & \\
1 mo (5) & 5 & 0 & 0 \\
2 mo (5) & 5 & 0 & 0 \\
3 mo (4) & 3 & 1 & 0 \\
6 mo (4) & 2 & 1 & 1 \\
12 mo (4) & 1 & 2 & 1 \\
CJSA & & & \\
1 mo (6) & 6 & 0 & 0 \\
2 mo (6) & 5 & 1 & 0 \\
3 mo (7) & 4 & 2 & 1 \\
6 mo (6) & 1 & 3 & 2 \\
12 mo (7) & 1 & 5 & 1 \\
\hline
\end{tabular}

At 3 months and earlier postoperatively, all the rabbits in the CJSC group and all the rabbits except for 1 in the CJSA group showed grade I-II pathologic changes that were reversible, and 1 rabbit in the CJSA group showed grade III changes that were between reversible and irreversible. After 3 months, 6 of 12 rabbits in the CJSC group and 6 of 20 rabbits in the CJSA group had grade I-II changes. Finally, 4 of 12 rabbits in the CJSC group and 10 of 20 rabbits in the CJSA group had grade III changes, and plexiform lesions (grade IV-VI), indicating irreversible PAH, were demonstrated in 2 of 12 rabbits in the CJSC group and 4 of 20 rabbits in the CJSA group; no irreversible pathologic changes were noted before 3 months in either group. CJSC, Common carotid artery and jugular vein shunt by cuff style method; CJSA, common carotid artery and jugular vein shunt by anastomosis.
Currently, several animal models can be used to study PAH. However, many of these models have limitations owing to surgical complications and difficulty, high mortality rates, and/or a failure to adequately mimic PAH in humans, including a failure to determine the exact point at which irreversible pathologic changes will occur. For example, owing to the complex operation protocol and high mortality rate, data from arteriovenous shunt models (representing hyperkinetic PAH) have seldom been reported. However, we confirmed in our earlier study ${ }^{17}$ that a successful hyperkinetic rabbit PAH model could be established using left common carotid artery and pulmonary trunk anastomosis. However, that method required a thoracotomy, which frequently resulted in death. In addition, a systemic-to-pulmonary artery shunt such as anastomosis of the left common carotid artery to the pulmonary trunk and others has resulted in a fairly high load to the pulmonary artery and heart, with the animals, especially the young, easily dying of heart failure. However, if the period after surgery was short, the PAH model was probably established unsuccessfully. Therefore, the success rate of that model was low. In response, we attempted to establish a novel hyperkinetic PAH model without thoracotomy and found that the pathophysiologic and histopathologic changes of the model were ultimately similar to those of congenital heart disease. This model can now serve as a platform for future PAH research, because it has both low mortality and a high success rate and adequately reflects the conditions present in humans.

Rats have also been commonly used for PAH models, along with sheep and dogs. ${ }^{16,21}$ However, studies using rabbits have rarely been reported. The blood vessels of the rats are too fine to perform anastomosis, consequently a shunt in a cuff style has typically been used. ${ }^{18,19} \mathrm{We}$ had previously established another PAH model using a left common carotid artery and left jugular vein shunt in a cuff style in Wistar rats; however, the long-term patency rate was questionable. ${ }^{18}$ Sheep, dogs, and other large animals have blood vessels suitable for anastomosis, but space and feeding constraints have made it difficult to house and feed a sufficient quantity of these animals for statistical analysis. In contrast, rabbits are excellent candidates for anastomosis and require less space and food. Therefore, our rabbit model will be a more economic model than those that use larger animals (eg, sheep). We believe rabbits provide a more appropriate model for hyperkinetic PAH research.

In our study, after 3 months, all the surviving rabbits with successful arteriovenous shunts had reached the standard of PAH. ${ }^{22}$ To exclude the influence of the arteriovenous shunt, we measured the pulmonary artery pressure before and after closure of the shunt vessels. We also found that the right ventricular hypertrophy indexes of the 2 model groups (CJSA and CJSC) were greater than those of the sham 


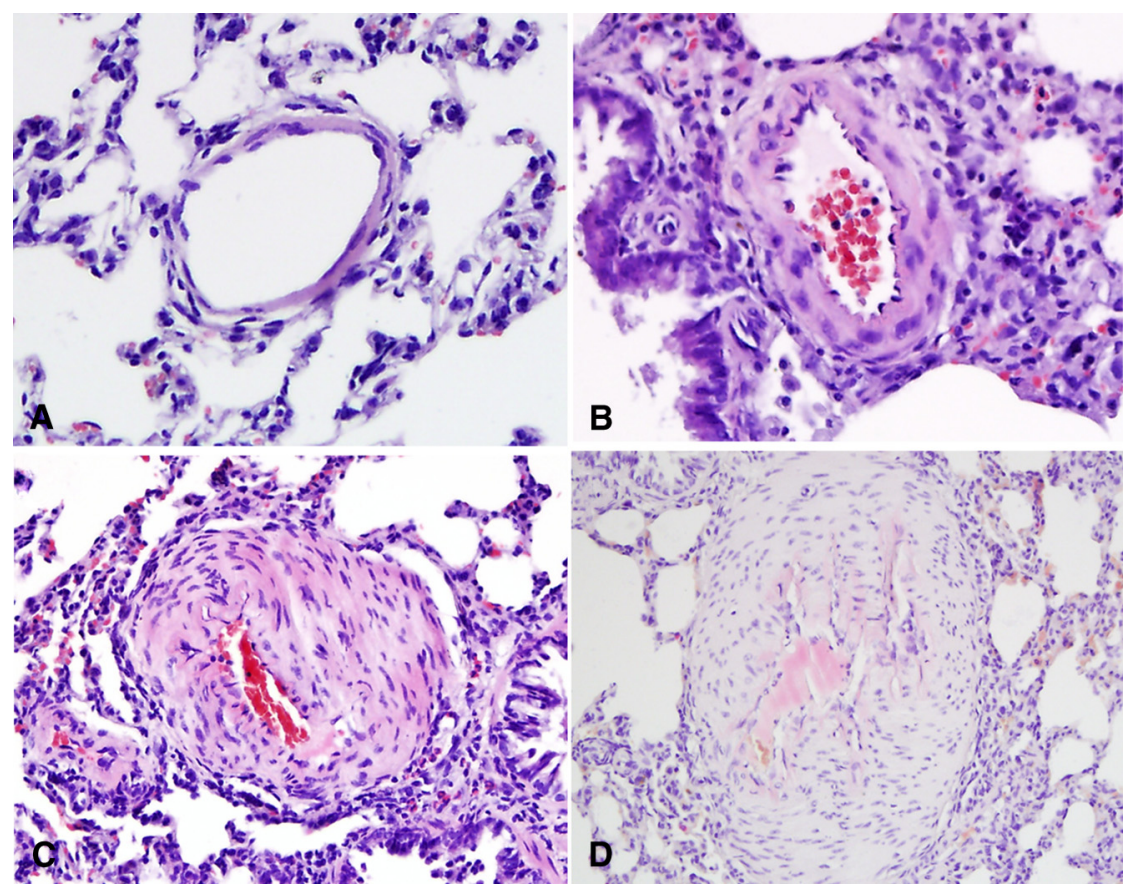

FIGURE 7. Pathologic changes in pulmonary artery postoperatively (hematoxylin-eosin stain, $\times 200$ ). A, Sham operation group; B, grade I to II intimal thickening and medial hypertrophy of arterioles; C, grade III advanced medial thickening and severe narrowing or occlusions of arterioles; and D, grade IV-VI plexiform lesions of arterioles.

operation group. Therefore, we have clearly demonstrated that a successful common carotid artery and jugular vein shunt was formed using either anastomosis or cuff techniques that ultimately led to pathologic features consistent with PAH.

It took longer to perform the arteriovenous anastomosis than the arteriovenous shunt in the cuff procedure. The longer operation time resulted from the general difficulty in inosculating fine vessels. The total period from anesthesia induction to the end of the operation was about $1.40 \pm 0.12$ hours in the CJSC group and $1.48 \pm 0.21$ hours in the CJSA group. However, as the proficiency with the anastomosis technique increased, the operative time for the CJSA group became shorter. One of the most important and difficult steps during both procedures was isolating the artery. It was easily damaged, which frequently led to bleeding. Some of the rabbits died because of this. In the CJSC group, the thin thread used to ligate the cuff could drop off easily, causing profuse bleeding and, typically, death within 24 hours of surgery. Therefore, the CJSC group had a greater mortality rate than the CJSA group. In addition to a greater mortality rate, the CJSC group had a lower patency rate. We would suggest 2 possible explanations for this. One explanation is that the internal diameter of cuff is not fit for the vessels of all the rabbits, such that blood flows from the wide vessel to the narrow cuff graft; thus, the wide vessel, despite the administration of aspirin, begins to occlude owing to thrombosis at the narrow site. Another possible explanation is that the cuff graft is recognized by the body as a foreign material, which can result in a chronic inflammatory reaction that could also lead to occlusion. Nonetheless, PAH was successfully formed 3 months postoperatively in both groups. However, the cuff graft will become relatively narrow owing to the growing artery. This can cause occlusions in the CJSC group, as previously described. In contrast, the arteriovenous anastomosis can grow in the CJSA group, reducing the complications stemming from differences in the vessel diameter of the individual rabbits.

To our knowledge, this is the first study to evaluate PAH using the Heath-Edwards classification system ${ }^{20}$ in rabbits at $1,2,3,6$, and 12 months after surgery, monitoring the pulmonary vascular pathologic changes as they progressed from reversible to irreversible. Understanding the kinetics of these changes will be indispensable in our future PAH research. For instance, we could study rabbits within 3 months of surgery to use a PAH model whose pulmonary vascular pathologic changes are reversible. This would be suitable for studying the effect of certain drugs or cytokines that might prevent or reverse vascular remodeling. In contrast, if we choose to study PAH using angiogenesis, we can work with animals $\geq 3$ months after surgery, at which time these changes will be permanent, allowing us to exclude influencing factors that might affect vascular remodeling. 


\section{CONCLUSIONS}

Hyperkinetic PAH can be established using a common carotid artery and jugular vein shunt in rabbits. However, the anastomosis method was superior to the cuff style method, with a greater patency rate, lower mortality rate, and greater overall success rate. Therefore, the CJSA method can provide a reliable, convenient, economic, and stable PAH model that represents the aberrant hemodynamic state in children with congenital heart disease. We can use this model to study the mechanisms of, and therapeutic approaches targeting, hyperkinetic PAH. In addition, we have established the point at which reversible or irreversible pathologic changes occur, offering 2 distinct models that can be selected according to the research directions in future studies on PAH.

\section{References}

1. Humbert M, Sitbon O, Chaouat A, Bertocci M, Habib G, Gressin V, et al. Survival in patients with idiopathic, familial, and anorexigen-associated pulmonary arterial hypertension in the modern management era. Circulation. 2010;122: 156-63.

2. Madani MM, Wittine LM, Auger WR, Fedullo PF, Kerr KM, Kim NH, et al. Chronic thromboembolic pulmonary hypertension in pediatric patients. J Thorac Cardiovasc Surg. 2011;141:624-30.

3. Drake JI, Bogaard HJ, Mizuno S, Clifton B, Xie B, Gao Y, et al. Molecular signature of a right heart failure program in chronic severe pulmonary hypertension. Am J Respir Cell Mol Biol. 2011;45:1239-47.

4. Beghetti M, Tissot C. Pulmonary hypertension in congenital shunts. Rev Esp Cardiol. 2010;63:1179-93.

5. Wang Y, Jing L, Zhao XM, Han JJ, Xia ZL, Qin SC, et al. Protective effects of hydrogen-rich saline on monocrotaline-induced pulmonary hypertension in a rat model. Respir Res. 2011;12:26.

6. Hirose K, Marui A, Arai Y, Kushibiki T, Kimura Y, Sakaguchi H, et al. Novel approach with intratracheal administration of microgelatin hydrogel microspheres incorporating basic fibroblast growth factor for rescue of rats with monocrotaline-induced pulmonary hypertension. J Thorac Cardiovasc Surg. 2008:136:1250-6

7. Suhara H, Sawa Y, Fukushima N, Kagisaki K, Yokoyama C, Tanabe T, et al. Gene transfer of human prostacyclin synthase into the liver is effective for the treatment of pulmonary hypertension in rats. J Thorac Cardiovasc Surg. 2002 123:855-61.

8. Nisbet RE, Bland JM, Kleinhenz DJ, Mitchell PO, Walp ER, Sutliff RL, et al. Rosiglitazone attenuates chronic hypoxia-induced pulmonary hypertension in a mouse model. Am J Respir Cell Mol Biol. 2010;42:482-90.

9. Mitani Y, Maruyama J, Jiang BH, Sawada H, Shimpo H, Imanaka-Yoshida K, et al. Atrial natriuretic peptide gene transfection with a novel envelope vector system ameliorates pulmonary hypertension in rats. J Thorac Cardiovasc Surg. 2008;136:142-9.

10. Zhao YY, Malik AB. A novel insight into the mechanism of pulmonary hypertension involving caveolin-1 deficiency and endothelial nitric oxide synthase activation. Trends Cardiovasc Med. 2009;19:238-42.

11. Nicolls MR, Mizuno S, Taraseviciene-Stewart L, Farkas L, Drake JI, A Husseini A, et al. New models of pulmonary hypertension based on VEGF receptor blockade-induced endothelial cell apoptosis. Pulm Circ. 2012;2:434-42.

12. Garcia R, Diebold S. Simple, rapid, and effective method of producing aortocaval shunts in the rat. Cardiovasc Res. 1990;24:430-2

13. Ciuclan L, Bonneau O, Hussey M, Duggan N, Holmes AM, Good R, et al. A novel murine model of severe pulmonary arterial hypertension. Am J Respir Crit Care Med. 2011;184:1171-82.

14. Jungebluth $P$, Ostertag $H$, Macchiarini P. An experimental animal model of postobstructive pulmonary hypertension. J Surg Res. 2008;147:75-8.

15. Pohlmann JR, Akay B, Camboni D, Koch KL, Mervak BM, Cook KE. A low mortality model of chronic pulmonary hypertension in sheep. J Surg Res 2012; 175:44-8.

16. Zhou X, Wang D, Castro CY, Hawkins H, Lynch JE, Liu X, et al. A pulmonary hypertension model induced by continuous pulmonary air embolization. J Surg Res. 2011;170:e11-6.

17. Wang W, Liu R, Cao G, Zhang F, Zhang Y, Zhang Z, et al. A reliable rabbit model for hyperkinetic pulmonary hypertension. J Thorac Cardiovasc Surg. 2010;140: 395-9.

18. Liu K, Liu R, Cao G, Sun H, Wang X, Wu S. Adipose-derived stromal cell autologous transplantation ameliorates pulmonary arterial hypertension induced by shunt flow in rat models. Stem Cells Develop. 2011;20:1001-10.

19. Li FH, Xia W, Li AW, Zhao CF, Sun RP. Inhibition of rho kinase attenuates high flow induced pulmonary hypertension in rats. Chin Med J. 2007;120:22-9.

20. Heath D, Edwards JE. The pathology of hypertensive pulmonary vascular disease: a description of six grades of structural changes in the pulmonary arteries with special reference to congenital cardiac septal defects. Circulation. 1958 18(4 Pt 1):533-47.

21. Luan Y, Zhang Z-H, Wei D-E, Lu Y, Wang Y-B. Effects of autologous bone marrow mononuclear cells implantation in canine model of pulmonary hypertension. Circulation. 2012;76:977-85.

22. Farber HW, Loscalzo J. Pulmonary arterial hypertension. N Engl J Med. 2004 351:1655-65. 